Cahiers $d u$ MONDE RUSSE

\section{Cahiers du monde russe}

Russie - Empire russe - Union soviétique et États indépendants

$60 / 4 \mid 2019$

Varia

\title{
Rebecca REICH, State of Madness. Psychiatry, Literature, and Dissent After Stalin
}

\section{Grégory Dufaud}

\section{OpenEdition}

\section{Journals}

Édition électronique

URL : https://journals.openedition.org/monderusse/11533

DOI : $10.4000 /$ monderusse. 11533

ISSN : $1777-5388$

\section{Éditeur}

Éditions de l'EHESS

\section{Édition imprimée}

Date de publication : 1 octobre 2019

Pagination : 865-867

ISBN : 978-2-7132-2797-4

ISSN : $1252-6576$

Référence électronique

Grégory Dufaud, «Rebecca REICH, State of Madness. Psychiatry, Literature, and Dissent After Stalin », Cahiers du monde russe [En ligne], 60/4 | 2019, mis en ligne le 01 octobre 2019, consulté le 06 janvier 2023. URL : http://journals.openedition.org/monderusse/11533; DOI : https://doi.org/10.4000/ monderusse. 11533 


\section{Rebecca REICH}

\section{State of Madness}

Psychiatry, Literature, and Dissent After Stalin

DeKalb : Northern Illinois University Press, 2018, 283 p.

Face à la multiplication des protestations en Union soviétique, l'un des moyens utilisés par la direction politique à partir des années soixante pour les contenir est de requérir les services des psychiatres légaux. Dans ce livre, Rebecca Reich examine comment des dissidents (dissenters) s'emploient, grâce à la littérature, à « résister à la pathologisation de l'inakomyslie [non-conformisme] » (p. 5). Par dissenter, elle entend un individu qui manifeste son désaccord vis-à-vis du régime en se plaçant délibérément en marge de la société : à la différence du dissident, le dissenter n'est donc pas forcément un activiste engagé contre l'État. Pareille définition lui permet de s'intéresser à des auteurs aux profils très variés tant en termes d'engagement politique que de production littéraire : Aleksandr Vol'pin, Vladimir Bukovskij, Semen Gluzman, Iosip Brodskij, Andrej Sinjavskij et Venedikt Erofeev. Jamais justifié, ce choix ne va pourtant pas de soi : qu'ont ainsi en commun le parcours et l'œuvre de Volpin avec ceux d'Erofeev?

Les dissidents dénoncent l'usage fait de la psychiatrie par la direction politique pour les réduire au silence. Pour eux, cet usage renvoie à la nature autoritaire de l'État soviétique et, de ce point de vue, leur analyse diffère de celle livrée au même moment par Michel Foucault pour qui il revient à l'État libéral et bourgeois d'avoir transformé la folie en un problème d'ordre public ${ }^{1}$. La thèse de l'ouvrage est que les dissidents considèrent la psychiatrie comme un « art » subjectif auquel ils opposent des moyens artistiques : empruntant à Mihail Bahtin, R. Reich avance qu'ils proposent, contre le discours monologique (une vision unique du monde) des psychiatres, un discours dialogique (une vision plurielle du monde). D'après elle, le monologisme du discours psychiatrique est similaire à celui du réalisme socialiste et cette similitude rend « la psychiatrie et la littérature d'État mutuellement intelligibles $\gg$ (p. 17). Par la dialogisation du discours psychiatrique, les dissidents réaffirment alors la différence entre la vie et l'art que le réalisme socialiste prétendait abolir.

Le premier chapitre montre en quoi la psychiatrie peut être assimilée à un « art ». Selon l'auteur, elle l'est à deux titres : non seulement par les techniques et les savoirs mobilisés, mais aussi par le caractère littéraire des écrits psychiatriques. Le diagnostic doit permettre la production d'un récit qui appréhende l'état du patient en resituant le trouble dans une progression temporelle tendue entre l'avant et l'après. Reposant sur l'observation du patient par le psychiatre et sur ses échanges avec lui, sa validité dépend de la capacité du psychiatre à conduire les échanges. Il a pour spécificité de convertir le dialogue entre le médecin et le patient en un monologue du médecin. En psychiatrie légale, la possibilité de réaliser un diagnostic rétrospectif est capitale pour déterminer la responsabilité du prévenu. Le diagnostic est subjectif par nature, et pourtant les psychiatres lui confèrent un caractère objectif. Dans la lutte contre les protestataires, le diagnostic a une dimension 
performative plutôt que constative : il transforme les dissidents en patients et le piège qu'il forme se referme un peu plus à chaque fois que le patient se proclame sain d'esprit. D'après R. Reich, les psychiatres se sont approprié le réalisme socialiste au niveau de la méthode d'abord, car les psychiatres se donnent pour mission de corriger le patient de la même manière que le réalisme socialiste ambitionnait de changer la société ; sur le plan de l'objet ensuite, puisque considérant les productions artistiques, ils associent les œuvres réalistes à des esprits sains et les œuvres expérimentales à la folie.

La nature subjective du diagnostic est ce qui autorise les dissidents à s'en emparer et à le manipuler. La littérature clandestine leur offre ainsi la possibilité de contester les conclusions de la psychiatrie légale. Le deuxième chapitre montre comment. Les dissidents signalent d'abord les équivoques de la psychiatrie : pour Vol'pin, le diagnostic relève moins de la science que d'un discours autoritaire et irrationnel. Ils utilisent ensuite les ambiguïtés du regard médical pour le renverser. L'anormalité est investie d'un nouveau sens : elle est synonyme de santé et de lucidité. Bukovskij et Gluzman appellent alors à soumettre les psychiatres à l'examen médical. Les dissidents se donnent finalement un rôle de réformateur : au titre de leur lucidité, dans une société poststalinienne amenée à évoluer, mais où les héritages sont si lourds, leur responsabilité doit être de conduire les changements.

Avec Brodskij, la littérature opère un autre renversement, étudié dans le troisième chapitre : pour lui, contrairement à la thèse marxiste selon laquelle « l'existence détermine la conscience », c'est la « conscience qui détermine l'existence ». Cette inversion se fonde sur la volonté de défamiliarisation (ostranenie) et de formation de ce sentiment d'étrangeté que Viktor Šklovskij avait mis au fondement de tout procédé créatif. Dans le poème Gorbunov et Gorčakov, où deux fous échangent dans un hôpital psychiatrique près de Leningrad, Brodskij suggère que l'intrication permanente de la conscience et de l'existence permet de préserver sa raison et d'exprimer un point de vue propre : le contrepoint dialogique est ainsi posé comme nécessaire à la défamiliarisation.

Ainsi que le montre le quatrième chapitre, cette défamiliarisation est également pensée par Sinjavskij comme un outil de création artistique. Celui-ci n'a toutefois pas la même finalité que chez Brodskij : pour Sinjavskij, elle vise à réintroduire la limite entre la vie et l'art que l'État ne cesse de vouloir confondre au titre de la théorie de la réflexion (teorija otraženija) qui, prolongeant la thèse selon laquelle « l'existence détermine la conscience », a donné naissance au réalisme socialiste. Sinjavskij et son alter ego Abram Terc sous le nom duquel il a signé plusieurs textes pensent ainsi la société comme un matériau, malléable, de production littéraire. Mais quand Siniavski insiste sur le caractère fictionnel de Terc, les juges, les critiques littéraires et les psychiatres estiment que la distinction entre les deux personnages n'est pas aussi claire qu'il l'affirme : pour eux, ce n'est là rien d'autre qu'un procédé destiné à échapper à ses responsabilités.

Ce rapport de l'art à la vie est au cœur du travail d'Erofeev qui interroge les différents sens attribués à la folie et auquel est consacré le dernier chapitre de l'ouvrage. La Nuit de Walpurgis est une pièce de théâtre qui se déroule dans un établissement 
psychiatrique où le principal protagoniste est interné pour dissidence. La folie et l'alcoolisme sont présentés comme la norme dans la société poststalinienne : « La santé mentale est devenue une pathologie, tandis que les catégories diagnostiques sont vidées de leur sens » (p. 202). Dans une société où la frontière entre la folie et la raison est brouillée, ne subsistent alors plus que la simulation et la dissimulation. Le théâtre engage ainsi un type de rapport à la réalité quand il n'interroge pas la réalité elle-même.

R. Reich propose une étude riche à la croisée de l'histoire politique, de l'histoire intellectuelle et de l'histoire littéraire qui offre un regard neuf sur la période poststalinienne et les agissements face à l'oppression. Mais ce beau livre se serait certainement montré plus incisif si l'auteur avait davantage resserré sa démonstration pour mieux faire ressortir ses idées : du fait de l'analyse parfois très fouillée des œuvres, on ne voit pas toujours immédiatement ce vers quoi R. Reich veut nous conduire.

1 - Michel Foucault, Histoire de la folie à l'âge classique, Gallimard, 1972.

Gregory Dufaud

Sciences Po Lyon 